\title{
One-step pipetting of barcoded planar microparticles into compact monolayer assembling chip for efficient readout of multiplexed immunoassay
}

Sangwook Bae ${ }^{I^{*}}$, Daewon Lee ${ }^{I^{*}}$, Hunjong $\mathrm{Na}^{2}$, Jisung Jang ${ }^{2}$ and Sunghoon Kwon ${ }^{1,2}$

\author{
${ }^{1}$ Department of Electrical and Computer Engineering, \\ Seoul National University, Gwanak-ro, Gwanak-gu, Seoul 151-744, South Korea \\ E-mail:skwon@snu.ac.kr
}

${ }^{2}$ QuantaMatrix Inc., Medical Innovation Center, Seoul National University Hospital, Seoul, 03080, Republic of Korea.

* these authors contributed equally to this work

\begin{abstract}
Barcoded planar microparticles have many qualities suitable for developing cost-efficient multiplexed immunoassays. But at the translational research level, there are a number of technical aspects yet remain to be addressed which includes robustness and efficiency of the assay readout process. Assay readout process involves automated barcode identification and signal intensity values from each planar microparticle. For this, each microparticle has to be correctly aligned for correct barcode readout while being, ideally, compactly assembled for maximum microparticle imaging efficiency. To simultaneously achieve such alignment and assembly of microparticles but in a straightforward manner, we designed a microfluidic microparticle assembling chip that only requires a single pipetting step. Our design utilizes capillary flow based guided particle assembly, which allows maximum microparticlebased immunoassay readout efficiency. With the aid of image processing algorithms, we obtained good multiplex immunoassay readout accuracy similar to conventional imaging platforms. Our approach is applicable to both soft elastomer materials (e.g. PDMS) and rigid materials (e.g. polystyrene), the latter of which is frequently used for injection molding based mass production. We anticipate our device could help developing facile and user-friendly platform technologies based on barcoded planar microparticles.
\end{abstract}

\section{Introduction}

To date, various suspension array-based assay platforms have been developed primarily to increase the multiplexity of assays. Among those, barcoded planar microparticles have many desirable qualities. First is scalability; the potential number of simultaneous measurement (multiplexity) grows proportionally with the number of encodable barcodes which is significantly higher than most nonbarcoded particles or assays ${ }^{1-6}$. Second is generality; due to many chemical modifications and particle material available ${ }^{7}$, these particles can be applied to detecting various analytes including oligonucleotides $^{8-13}$, proteins ${ }^{14-18}$ and cells ${ }^{19-22}$. And last is affordability; adaptation of sensitive colorimetric assays ${ }^{23,24}$ and inexpensive readout tools such as scanners or smartphones, ${ }^{4,25,26}$ enables development of compact, facile devices. Therefore, one could rationally argue barcoded planar microparticles are good candidates for developing highly multiplexed immunoassays in an efficient manner.

Although many proof-of-concept studies were done, many still require companion technologies to enable translation to commercial products ${ }^{27}$. In one respect, to make such multiplexed immunoassay simple and efficient, two important yet not well-appreciated steps have to be dealt with; i) efficiently 
aligning the particles and ii) tightly packing these aligned particles into a single layer on a flat surface. This is because, since these microparticles have planar barcodes, accurate and reproducible reading requires particle alignment. Also, tightly packing these aligned particles is necessary to maximize reading throughput.

To address these particle maneuvering problems (aligning and packing), several methods have been invented including magnetic ${ }^{1}$ fluidic flow-guided ${ }^{28-33}$ and shape-guided ${ }^{22,33}$ approaches. One commercialized platform that utilizes microfluidic flow-guided assembly is Mycartis now available for clinical and pharmaceutical applications (patent EP2684605A1). Such fully automated microfluidic device will find many needs in hospital labs and pharmaceutical companies where routine, large scale immunoassay is necessary.

But one could also anticipate need for multiplexed immunoassays from other research labs or pointof-care device developers. For this, further simplifying these particle maneuvering mechanisms to something plain as pipetting would be desirable. We previously developed a method for one-step pipetting-based alignment and assembly of barcoded planar particles ${ }^{34,35}$. Although this facile method have great potential for multiplexed chemical reaction screening, the packing density had to be sacrificed to prevent well-to-well cross-reaction.

Here, we present a new chip for simultaneous alignment and packing of barcoded planar microparticles. A single particle pipetting of these particles into the chip leads to their automatic alignment and monolayer packaging into the field of view (FOV) of $2 x 2.5 \mathrm{~mm}$ with maximum density. The mechanism doesn't need any syringe pumps, controllers or other exterior devices. Users can efficiently obtain multiplexed immunoassay results from the packed particles. Unlike spreading particles on plain surfaces, our chip based method doesn't suffer from particle clumping, overlapping or density fluctuation. We applied our device to fluorescence based multiplexed cytokine immunoassay and obtained equivalent results to traditional planar assay. Although our initial chip design was based on PDMS, we obtained similar particle packing performances with polystyrene (PS) chips, which is potentially better suited for scale up production and commercialization.

\section{Materials and Methods}

\section{Materials}

PDMS, SU8 2015, SU8 2050, isopropyl alcohol (IPA), and SU8 developer were purchased from Microchem. Perfluorooctyltrichlorosilane, tetraethoxyl silane (TEOS), Bovin serum albumin (BSA), (3-aminopropyl)triethoxysilane (APTES) $>98 \%$, Succinic anhydride (SA) $>99 \%$, Polyurethane (PUA), 3-(Trimethoxysilyl)propyl methacrylate (TMSPA), Trimethylolpropane ethoxylate triacrylate (ETPTA), 2-Hydroxy-2-methylpropiophenone (Darocur), and Triethylamine (TEA) $>99.5 \%$ were purchased from Sigma Aldrich. Other chemicals and solvents including N-Hydroxysulfosuccinimide Sodium (NHS) salt 97\%, Absolute ethanol, NN-Dimethylformamide (DMF), and 1-(3-Dimethylaminopropyl)-3ethylcarbodiimide hydrochloride (EDC) $98+\%$ were purchased from Alfa Aesar. Capture antibodies, biotin-labelled polyclonal antibodies, and antigens were purchased from either Millipore, eBioscience, or BD Bioscience. Streptavidin-linked phycoerythrin (SA-PE) was purchased from ProZyme. [2-(Nmorpholino)ethanesulfonic acid] (MES) buffered saline pack was purchased from Thermo Scientific. Chemicals sensitive to moisture, such as NHS and EDC, was stored in desiccators. Other reagents were stored according to the manufacturer's recommendation.

\section{Chip synthesis}

For the PDMS chips, we designed FCG type film masks or chrome masks with Autocad and manufactured by Microtech. On 4 inch silicon wafers, for various SU8 pattern heights, we performed spin coating, soft baking, UV exposure, post-exposure baking was done as the manufacturer's guide. After developing, hard baking was done at $150^{\circ} \mathrm{C}$ for 10 minutes. To reduce the surface energy, we coated the SU8 patterned wafer with perfluorooctyltrichlorosilane by dispensing few microliters of perfluorooctyltrichlorosilane on slide glasses heated to $85^{\circ} \mathrm{C}$ and placing the wafer over the slideglass 
bioRxiv preprint doi: https://doi.org/10.1101/2022.01.03.474850; this version posted January 4, 2022. The copyright holder for this preprint

for 1 minute. PDMS (Sylgard 184, DowCorning) was mixed with curing agent at $10: 1 \mathrm{w} / \mathrm{w}$ and poured into the SU-8 mold. After baking at $110^{\circ} \mathrm{C}$ for 30 min,the PDMS pattern was peeled off and attached to a slide glass by $\mathrm{O} 2$ plasma treatment (CUTE-MP, FemtoScience).

For PUA chip synthesis, we spread liquid PUA on a glass mold with a ramp with varying angle then UV cured the liquid into a polymer matrix. Then we assembled these triangular PUA blocks on the SU8 pattern of the original particle packing channel. Using this modified 3-dimensional pattern, we fabricated a series of PDMS molds and a final PUA channel that has a channel inlet height gradient.

For PS chip, we outsourced the injection molding to Quantamatrix using our autocad design for the optimized gradient channel design obtained from PUA chip experiments.

\section{Particle synthesis}

We used the previously reported protocol ${ }^{36}$. Briefly, ETPTA was mixed with $10 \%$ w/w TMSPA and Darocur was added at 10\% w/w of the ETPTA/TMSPA mixture. the ETPTA/TMSPA/Darocur mixture was poured on air plasma treated hydrophilic glass slides. Using masks for contact printing UV lithography using negative resist was performed. TEOS was used for silica coating. Amine groups were coupled to the particle surface using $0.095 \mathrm{~g} / \mathrm{mL}$ APTES solution (in absolute ethanol) and stirred at $25^{\circ} \mathrm{C}$ for 2 hours. Bead were washed with absolute ethanol and dried at $110^{\circ} \mathrm{C}$ for 10 minutes. Terminal amine carboxylation was done by adding carboxylation solution $(6 \mathrm{mg} \mathrm{SA}, 8.4 \mathrm{uL}$ of $7.2 \mathrm{M}$ TEA in $1 \mathrm{ml}$ DMF) and stirring at $25^{\circ} \mathrm{C}$ for 2 hours. Beads were washed with DMF, absolute ethanol, and MES buffered saline. EDC/NHS-cross-linking solution (5mg NHS, 5mg EDC per $1 \mathrm{ml} \mathrm{MES}$ ) was then added and stirred at room temperature for 25minutes and washed with MES. Capture antibodies in MES $(14 \mathrm{ug} / \mathrm{mL})$ was mixed with bead and stirred (1200rpm) at room temperature for $\mathbf{2}$ hours. After antibody cross-linking, the beads were blocked with BSA solution (1\% BSA in PBS).

\section{Immunoassay}

Immunoassay protocol was adapted from a previous report (Jiyun Kim et al.,). Particles were incubated with antigens (diluted in $0.1 \%$ BSA in PBS) and stirred at $4^{\circ} \mathrm{C}$ for 2 hours. After washing with washing solution ( $0.1 \%$ BSA, $0.02 \%$ Tween 20 in PBS), particles were incubated with $2 \mu \mathrm{g} / \mathrm{ml}$ biotin-labelled anti-antigen polyclonal antibodies prepared in dilution solution (PBS solution containing $0.1 \% \mathrm{BSA}$ ) at $650 \mathrm{rpm}, 25^{\circ} \mathrm{C}$ for 1 hour and were washed with washing solution. Finally, particles were incubated with SA-PE solution (1 $\mu \mathrm{g} / \mathrm{ml} \mathrm{SA}$-PE prepared in dilution solution) for $30 \mathrm{~min}$ and were washed rigorously with washing solution before imaging. We imaged the particles with Olympus fluorescence microscope ( $4 \times$ objective lens) with emission wavelength filtered at $575 \mathrm{~nm}-625 \mathrm{~nm}$ at exposure time of $0.2 \mathrm{sec}$. The immunoassay readouts from either the packing channel or 96wellplate wells were decoded using an in-built program from Quantamatrix Inc.

\section{COMSOL simulation}

We used COMSOL Multiphysics 5.4 for our fluidic simulations. We used the 'particle tracing for fluid flow' module with no wall slip condition, extra-coarse meshing, and creeping normal inflow with velocity of $3.5 \mathrm{~mm} / \mathrm{sec}$. Parameter sweeping was used for either i) sweeping the effects of the parameter (e.g. channel height or gradient angle variation) on particle movements or ii) determining the optimal diverging and converging channel design. 


\section{Results}

\section{- Channel design optimization}

To assess the basic characteristic of planar particle dispersion, we initially tested simple particle dispersion on 96 wellplate wells. The goal was to obtain as many particles per FOV possible while minimizing any unwanted dispersion patterns like particle overlapping and particle tilting. By testing various particle concentration, we observed the trade-off between particle packing density and particle monolayer assembly rate (Fig 2c). To overcome this trade-off, we had to come up with a guided assembly method that enables both particle alignment and dense packing. For this we designed a fluidic channel with particle stopping pillars (Fig 1.).

With dummy planar particles, we first tested different PDMS channel heights. Our particles were planar cylinders with 100um in diameter and 30um in thickness and were suspended in DIW. Our initial intuition was that channel height should be bigger than the particle height because the suspended particles will have random orientation and thus will need sufficient space to rotate towards proper alignment while moving towards the particle packing region of the channel. However, with increasing channel height, the packed particles will occasionally overlap with adjacent particles which is detrimental to proper signal readout. Our experiment results showed that the channel height has to be nearly identical to the particle thickness (Fig S1.) Only then, we were able to get a $99.7 \%$ monolayer packing efficiency. The reason these planar particles with 100um diameter were able to enter the 30um height channel was due to the flexible bulging of the PDMS channel (Fig S2a). In other words, when we inserted the pipette into the channel inlet and inject the particle-suspended solution, the pressure created a channel height gradient from $>100 \mathrm{um}$ at the inlet gradually decreasing to $31 \mathrm{um}$ at the particle packing region. Harnessing this channel height gradient became the key design factor for our particle packing channel.

With this initial PDMS channel design, we created a chip with 8 parallel channels to increase readout throughput (Fig 2a). The channels were arranged in a way that the particle packing regions were aligned in a single line thus enabling easy sequential imaging. We dispensed approximately 600 to 700 particles per channel using normal pipettes. When compared simple particle dispensing in 96wellplates, our PDMS channel was superior in particle packing density as well as monolayer packing efficiency (Fig 2b, c). Even with 3500 particle input, 96well results showed 3 times lower number of particles per FOV compared to PDMS channel with 700 particle input. And PDMS channel results showed almost complete monolayer assembly of the particles with only one or two particles per FOV lost during analysis due to particle overlapping. We also compared the particle imaging speed between our channel and 96wellplate dispensing. We designated imaging speed as simply the number of monolayer particles per FOV because the delay between each FOV imaging is comparable between our channel-based method and simple dispensing. Our channel design had one anticipated advantage in speed over simple dispensing due to the straight-line scanning mode rather than the raster scanning of $6 \sim 102 \mathrm{~mm} \times 2.5 \mathrm{~mm}$ FOVs per well $(\mathrm{d}=\sim 6.4 \mathrm{~mm}$ ) required to image circular wells. Overall, the image acquisition speed of our channel design was 18 30 times faster than 96 wellplate due to the significantly reduced number of image acquisition required.

\section{- Multiplexed immunoassay readout}

We then analyzed the multiplexed immunoassay readout capability of our channel design. We first created standard curves for both our particle packing channel and 96wells using barcoded particles for detecting IL-1 $\beta$. We treated our barcoded particles (single barcode) with varying concentration of IL-1 $\beta(0,800,4000,20000,100000 \mathrm{pg} / \mathrm{mL})$ and detection antibodies conjugated with fluorescent dyes. For each IL-1 $\beta$ concentration condition, particles were either injected into our channel or dispensed in 96 wells and imaged with a fluorescence microscope. The barcodes and corresponding fluorescence signal of each particle was decoded using a custom software created by Quantamatrix. The standard curves were identical between two particle packing methods (Fig 3a). We then performed 4plex immunoassay of cytokines. We prepared 4 types of barcoded particles with each having capture antibodies against IL-2, IL-4, IL-6, IL-5 respectively. We treated these particles with a mixture of cytokines with pre-determined concentrations; $10000 \mathrm{pg} / \mathrm{mL}$ for IL-2, 2000pg/mL for IL-4, $400 \mathrm{pg} / \mathrm{mL}$ 
for IL-5 and $0 \mathrm{pg} / \mathrm{mL}$ for IL-6. We then treated these particles with detection antibodies conjugated with fluorescent dyes. These particles were then either injected into our particle packing channel or dispensed on 96wellplates. Similar to the singleplex experiment, the assay result was overall comparable between 96wellplate and our particle packing channel (Fig S3a). However, our particle packing channel had a slight tendency of reduced signal at high antigen concentration (IL-2 and IL-4) while producing increased signal at low antigen concentration (IL-5 and IL-6). Most critically, our particle packing channel induced a false positive signal for the negative control (IL-6).

\section{- Correction of fluorescence profile obtained from particle packing channel}

We analyzed this discrepancy between 96 well and particle packing chip experiments. After screening each particle images decoded by the software, we noticed that when particles with low signal are placed adjacent to high signal particles, signal overestimation occurred in the low signal particle (Fig 3b, c). And the magnitude of such signal overestimation correlated with the number of adjacent high signal particles (Fig S3b). So we concluded the cause of such signal overestimation was fluorescence crossover between adjacent particles. To remove such errors, we introduced imaging processing techniques for error filtering (Fig 3d). We first assessed the effect of reducing the mask size from $100 \%$ down to $80 \%$ and $60 \%$ of the original mask radius. The mask size reduction had a slight effect of reducing the overestimated signals for low signal particles. We then reanalyzed the 96well and particle packing chip experiment images using these modified mask sizes. Although we reduced the signal overestimation for low signal particles in the packing channel, it was still higher than the 96well results. Then, to remove the image background signals, we masked the particles, measured the background pixel intensities, removed pixels with intensity above the median value, calculated the average of the remaining pixels, and subtracted this average value from all particle intensity values. We also assessed the effect of guided subsampling of particles. This was introduced to remove particles that have adjacent particles with high signal particles. Due to limited sample size, this subsampling method was done heuristically. But future large collection of images will help establish more objective and statistically defined thresholds for this process. We found that by applying all three filtering procedures above, the assay profiles between packing chip and 96wells becomes comparable. Most importantly, signal overestimation of the low signal particles in packing chips was successfully removed (fig 3e). By applying the three filtering steps above to our previous 4 plex immunoassay result, we were able to obtain similar assay profile between our packing chip and 96well experiments (Fig $\mathbf{4 c}, \mathbf{d})$. It should be noted that for the IL-1 $\beta$ standard curve generation experiments, unlike singleplex experiments in Figure 3a requiring 5 independent experiments required, the standard curve in Figure $4 \mathrm{c}$ was obtained by a single experiment using 5 plex assay.

\section{- Particle packing chip design with PUA and PS}

We then decided to design a particle packing chip that would be suitable for industrial manufacturing such as injection molding. As mentioned earlier, the channel height gradient design had to be applied. To determine the effective channel height gradient design, we tested a number of approaches. First we tested PUA-based channels having stepwise channel height reduction. Although our fluidic simulation predicted no significant turbulence (Fig S4a), the particle packing experiments with this design ended up with particle clogging at the channel height varying junctions (Fig S2b, c). COMSOL simulation showed that replacing the stepwise channel height reduction with a continuous height reduction (i.e. gradient channel height) could reduce the particle clogging problem (Fig 5a, b). So we tested gradient channel height designs with different gradient angles from $0.5^{\circ} \sim 10^{\circ}$. We empirically determined that the gradient angle have to be smaller than $2.5^{\circ}$ to effectively prevent particle clogging (Fig S2b, c). We also found that the width of the channel have to be wider at the end of the channel height gradient to prevent particle clogging (data not shown). To obtain further intuitions for the optimum channel design, we performed COMSOL fluidic simulation with parameter sweeping setup for the width of the end of the gradient channel. Using the particle tracing module we found that a certain diverging-converging channel width structure was needed to prevent particle clogging while minimize dead volume (Fig 5c, Fig S4b). In other words, sufficient divergence was needed to prevent particle clogging (or particle sticking to channel wall) while excess divergence leads to dead channel volume where no particles pass through. Based on this optimized design, we then created a mold for PS injection molding. The resulting 
particle packing PS chips were tested with fluorescent barcoded particles and were successfully imaged (Fig 5d, e). The barcodes were clearly readable by human eye even though the transparency of the PS chips were poor compared to PDMS channels. However, the particle decoding programs were not compatible with these images due to the frequent defects on the surface of these PS chips. Future optimization of these injection molding method will be required to reduce these channel defects.

\section{Conclusion}

We created a particle packing chip that is capable of simultaneous particle alignment and monolayer packing of non-spherical, planar microparticles. Performing barcoded microparticle immunoassay with our packing chip, compared to conventional wellplate, leads to 18 30 times faster assay readout with minimal data loss occurring from improper particle orientation. Other than the chip and a pipette, our strategy doesn't require cumbersome syringe pumps or other fluidic controlling modules. We expect this chip design will find utility in point-of-care applications where tools are encouraged to be simple yet high throughput, multiplexing capacity is required. 


\section{References}

1. Lee H, Kim J, Kim H, Kwon S. Colour-barcoded magnetic microparticles for multiplexed bioassays. Nat Mater., 9(9), 745-749, (2010).

2. Pregibon DC, Toner M, Doyle PS. Multifunctional encoded particles for high-throughput biomolecule analysis, Science, 315(5817), 1393-1396, (2007).

3. Bong KW, Chapin SC, Doyle PS. Magnetic Barcoded Hydrogel Microparticles for Multiplexed Detection, Langmuir,. 26(11), 8008-8014 (2010).

4. Bae, H. J. et al. Biomimetic microfingerprints for anti-counterfeiting strategies. Adv. Mater., 27, 2083-2089, (2015).

5. Hyung Jong Bae, Sangwook Bae, Jinsik Yoon, Cheolheon Park, Kibeom Kim, Sunghoon Kwon, Wook Park, Self-organization of maze-like structures via guided wrinkling, Science Advances, (2017)

6. Cheolheon Park, Hyung Jong Bae, Jinsik Yoon, Seo Woo Song, Yunjin Jeong, Kibeom Kim, Sunghoon Kwon*, and Wook Park, Gradient-Wrinkled Microparticle with Grayscale Lithography Controlling the Cross-Linking Densities for High Security Level Anti-Counterfeiting Strategies, ACS Omega, 6, 3, 2121-2126, (2021).

7. Le Goff, G.C., Srinivas, R.L., Hill, W.A., and Doyle, P.S., Hydrogel microparticles for biosensing, Eur. Polym. J., 2015.

8. Pregibon DC, Doyle PS. Optimization of Encoded Hydrogel Particles for Nucleic Acid Quantification. Anal Chem. 2009; 81(12):4873-4881.10.1021/Ac9005292 [PubMed: 19435332]

9. Choi NW, Kim J, Chapin SC, Duong T, Donohue E, Pandey P, et al., Multiplexed detection of mRNA using porosity-tuned hydrogel microparticles, Anal Chem., 84(21), 9370-9378, (2012).

10. Chapin SC, Appleyard DC, Pregibon DC, Doyle PS. Rapid microRNA Profiling on Encoded Gel Microparticles., Angew Chem Int Ed., 50, 10, 2289-2293, (2011).

11. Yeongjae Choi, Hyung Jong Bae, Amos C. Lee, Hansol Choi, Daewon Lee, Taehoon Ryu, Jinwoo Hyun, Seojoo Kim, Hyeli Kim, Suk-Heung Song, Kibeom Kim, Wook Park, Sunghoon Kwon, DNA Micro-Disks for the Management of DNA-Based Data Storage with Index and WriteOnce-Read-Many (WORM) Memory Features, Advanced Materials, 32, 37, (2020).

12. Lee, H., Srinivas, R.L., Gupta, A. and Doyle, P.S.,. Sensitive and Multiplexed On-chip microRNA Profiling in Oil-Isolated Hydrogel Chambers, Angew. Chem. In. Ed., 54, 2477-2481, (2015). 13. Lee, H., Shapiro, S., Chapin, S., and Doyle, P.S. "Encoded hydrogel microparticles for sensitive and multiplex microRNA detection directly from raw cell lysates," Anal. Chem., 88, 30753081, (2016).

14. David C. Appleyard, Stephen C. Chapin, and Patrick S. Doyle, Multiplexed Protein Quantification with Barcoded Hydrogel Microparticles, Anal. Chem., 83, 193-199, (2011).

15. Appleyard DC, Chapin SC, Srinivas RL, and Doyle PS. Bar-coded hydrogel microparticles for protein detection: synthesis, assay and scanning. Nat Protoc. 6(11),1761-1774, (2011).

16. Srinivas RL, Chapin SC, and Doyle PS. Aptamer-Functionalized Microgel Particles for Protein Detection. Anal Chem., 83(23),9138-9145, (2011).

17. Dong Yoon Oh, Hunjong Na, Seo Woo Song, Jinhyun Kim, Hyunsoo In, Amos Chungwon Lee, Yunjin Jeong, Daewon Lee, Jisung Jang, and Sunghoon Kwon, ELIPatch, a thumbnail-size patch with immunospot array for multiplexed protein detection from human skin surface, Biomicrofluidics, (2018).

18. Hyun Jee Lee, Yoon Ho Roh, Hyeon Ung Kim, Sun Min Kim and Ki Wan Bong, Multiplexed immunoassay using post-synthesis functionalized hydrogel microparticles, Lab Chip, 19, 111, (2019).

19. Tan, W.S., Lewis, C., Horelik, N., Pregibon, D., Doyle, P.S., and Yi, H., "Hierarchical Assembly of Viral Nanotemplates with Encoded Microparticles via Nucleic Acid Hybridization", Langmuir, 24, 12483-12488, (2008).

20. Lewis, C., Lin, Y., Yang, C., Manocchi, A., Yuet, K.P., Doyle, P.S., and Yi, H., "Microfluidic Fabrication of Hydrogel Microparticles Containing Functionalized Viral Nanotemplates", Langmuir, 26, 13436-13441, (2010).

21. Sangkwon Han, Hyung Jong Bae, Su Deok Kim, Wook Park, Sunghoon Kwon, An encoded 
viral micropatch for multiplex cell-based assays through localized gene delivery, Lab Chip, 14, (2017). 22. Daewon Lee, Amos Chungwon Lee, Sangkwon Han, Hyung Jong Bae, Seo Woo Song, Yunjin Jeong, Dong Yoon Oh, Seongkyu Cho, Junhoi Kim, Wook Park, and Sunghoon Kwon, Hierarchical shape-by-shape assembly of microparticles for micrometer-scale viral delivery of two different genes, Biomicrofluidics, 2018

23. Lee, H., Lee, J., Lee, S.G. and Doyle, P.S. Hydrogel-based colorimetric assay for multiplexed microRNA detection in a microfluidic device, Anal. Chem., 92, 5750-5755, (2020).

24. Juthani, N. and Doyle, P.S.,A Platform for Multiplexed Colorimetric microRNA Detection using Shape-encoded Hydrogel Particles, Analyst, 145, 5134-5140, (2020).

25. Gustav Svedberg, Yunjin Jeong, Hunjong Na, Jisung Jang, Peter Nilsson, Sunghoon Kwon, Jesper Gantelius and Helene Andersson Svahn, Towards encoded particles for highly multiplexed colorimetric point of care autoantibody detection, Lab Chip, 3, (2017).

26. Jiseok Lee, PaulW. Bisso, Rathi L. Srinivas, Jae Jung Kim, Albert J. Swiston and Patrick S. Doyle, Universal process-inert encoding architecture for polymer microparticles, Nat Mat., 13, 524529, (2014).

27. Gaelle C. Le Goff, Rathi L. Srinivas, W. Adam Hill, and Patrick S. Doyle, Hydrogel microparticles for biosensing, Eur. Polym. J., 72, 386-412, (2015).

28. Choi D, Jang E, Park J, and Koh WG, Development of microfluidic devices incorporating nonspherical hydrogel microparticles for protein-based bioassay, Microfluid Nanofluid., 5(5), 703-710, (2008).

29. Gerver RE, et al. Programmable microfluidic synthesis of spectrally encoded microspheres, Lab Chip, 12(22), 4716-4723, (2012).

30. Stephen C. Chapin, Daniel C. Pregibon and Patrick S. Doyle, High-throughput flow alignment of barcoded hydrogel microparticles, Lab Chip, 9, 3100-3109, (2009).

31. Lim, E.J., Ober, T.J., Edd, J.F., Desai, S.P., Neal, D., Bong, K.W., Doyle, P.S., McKinley, G.H., and Toner, M., Inertio-elastic Focusing of Bioparticles in Microchannels at Ultra-high Throughput, Nat. Commun., 5, 4120, (2014).

32. Eral, H.B., Safai, E.R., Keshavarz, B., Kim, J.J., Lee, J., and Doyle, P.S., Governing principles of alginate microparticle synthesis with centrifugal forces, Langmuir, 32, 7198-7209, (2016).

33. Kim, J.J., Chen, L., and Doyle, P.S., Microparticle Parking and Isolation for Highly Sensitive MicroRNA Detection, Lab Chip, 17, 3120-3128, (2017).

34. Eun Chung S, Kim J, Yoon Oh D, Song Y, Hoon Lee S, Min S, et al. One-step pipetting and assembly of encoded chemical-laden microparticles for high-throughput multiplexed bioassays. Nat Commun., 510.1038, (2014).

35. Seo Woo Song, Su Deok Kim, Dong Yoon Oh, Yongju Lee, Amos Chungwon Lee, Yunjin Jeong, Hyung Jong Bae, Daewon Lee, Sumin Lee, Jiyun Kim*, and Sunghoon Kwon, One-Step Generation of a Drug-Releasing Hydrogel Microarray-on-a-Chip for Large-Scale Sequential Drug Combination Screening, Adv. Sci., 6, 3, (2018).

36. Lily Nari Kim, Mira Kim, Keumsim Jung, Hyung Jong Bae, Jisung Jang, Yushin Jung, Jiyun Kim and Sunghoon Kwon, Shape-encoded silica microparticles for multiplexed bioassays, Chem. Commun., 60, (2015).

37. Jiyun Kim et al., Fiber composite slices for multiplexed immunoassays, Biomicrofluidics, (2015) 


\section{Figure Legends}

Figure 1. Schematic of a microfluidic, pipetting-based packing of encoded microparticles. Efficient readout of encoded microparticle-based multiplex immunoassay can be done by a pipetting-induced capillary flow-based monolayer assembly of microparticles into a microfluidic chip. The assay readout becomes efficient compared to simple microparticle dispensing on flat surface (i.e. 96 wellplate) because of simultaneous increase of microparticle assembly density and alignment quality. Scale bars are $100 \mu \mathrm{m}$.

Figure 2. Maximization of imaging throughput using parallel packing channels. (a) Schematic of microparticle assembly and 1D assay readout using an 8 parallel channel chip. After 8 microparticle pipetting into the channel inlets, assay readout can be done in an efficient 1D scanning motion due to the 8 FOVs being aligned in a straight line. (b) Example images of 8 FOVs containing packed microparticles. Scale bars are $200 \mu \mathrm{m}$. (c) Comparison of particle packing performances between 96 wellplate (well) and particle packing chip. For the 96wellplate experiments, we dispensed either 500, $700,1000,1800,2200$, or 3500 microparticles per well. For the packing chip experiment, we pipetted on average 600 microparticles per chip. perFOV (red): number of particles present in a single FOV. Monolayer (blue): number of microparticles per FOV that are assembled into a monolayer without particle overlapping or misaligned. Error bars indicate standard deviation.

Figure 3. Denoising the fluorescence crossover occurring in packed microparticles. (a) Standard curve of IL-1 $\beta$ immunoassay obtained from imaging singleplex immunoassay microparticles placed on either 96wellplates or in microparticle packing chips. Different code indicated different input IL-1 $\beta$ antigen concentration. Scale bar is $100 \mu \mathrm{m}$ (b) Bright field (upper panel) and fluorescence (lower panel) images of an example multiplex immunoassay readout, where different encoded microparticles showing different fluorescence intensity (or input antigen concentration). Red circles indicate low fluorescence intensity microparticles surrounded by high intensity microparticles. Blue circles indicate low fluorescence intensity microparticles not surrounded by high intensity microparticles. Scale bars are $100 \mu \mathrm{m}$. (c) Average fluorescence intensities of the microparticles indicated in (b). Error bars are standard deviations. (d) Fluorescence intensity filtering strategy for low fluorescence intensity microparticles surrounded by high intensity microparticles. Scale bars are $50 \mu \mathrm{m}$ for images in filter 1 and $200 \mu \mathrm{m}$ for filter 2. (e) Assessment of filter combinations on fluorescence intensity correction. The microparticle fluorescence intensity values obtained from 96wellplate experiments (but with additional application of filter 1 and 2) was used as a true value reference.

Figure 4. High throughput multiplexed immunoassay using packing chip. (a, b) Bright field (left panel) and fluorescence (right panel) images of a 4plex cytokine immunoassay using microparticle packing chips. As indicated in (b), each microparticles were correctly captured by our image processing algorithm. Scale bars are $400 \mu \mathrm{m}$ (a) or $200 \mu \mathrm{m}$ (b). (c,d ) Comparison of 5plex one-pot generated standard curve of IL-1 $\beta$ (c) and 4plex cytokine detection profiles (d) obtained by either 96wellplate experiment (blue) or microparticle packing chip (red). Input antigen concentrations were $10000 \mathrm{pg} / \mathrm{mL}$ for IL-2 (code 1), 2000pg/mL for IL-4 (code 2), 400pg/mL for IL-5 (code 4) and 0pg/mL for IL-6 (code 
3). Error bars are standard deviations.

Figure 5. Geometrical optimization of commercializable high stiffness material based fluidic channel. (a) Design of a solid, injection-molded, polystyrene, microparticle packing chip. The diverging and converging channel width and continuously reducing channel height was the key features to enable microparticle packing in such solid-sate microfluidic chips. (b) Simulation result comparing the microparticle channel adhesion density for various channel designs; the channel height profile of these designs were either a step-wise reduction or a continuous reduction with a certain gradient angel. Microparticle channel adhesion density indicates the number of microparticles adhere to the channel wall per unit time per unit area; a parameter we used to assess the microparticles' channel clogging effect, which must be minimized for efficient microparticle assembly. (c) Simulation result comparing channels with different channel width profiles on the microparticle dispersion efficiency. Color scheme indicates fluidic flow velocity. Each dot represents a microparticle. W indicates the maximum channel width. (d, e) Bright field (upper panels) and fluorescence (lower panels) images of multiplex immunoassay microparticles assembled in a PS microparticle packing chip. Scale bars are $400 \mu \mathrm{m}$ (d) and $200 \mu \mathrm{m}(\mathrm{e})$. 
bioRxiv preprint doi: https://doi. org/10.1101/2022 01.03 474850; this version posted January 4, 2022. The copyright holder for this preprint (which was not certified by peer review) is the author/funder, who has granted bioRxiv a license to display the preprint in perpetuity. It is made available under aCC-BY-NC-ND 4.0 International license.

\section{Figures}

Figure 1.
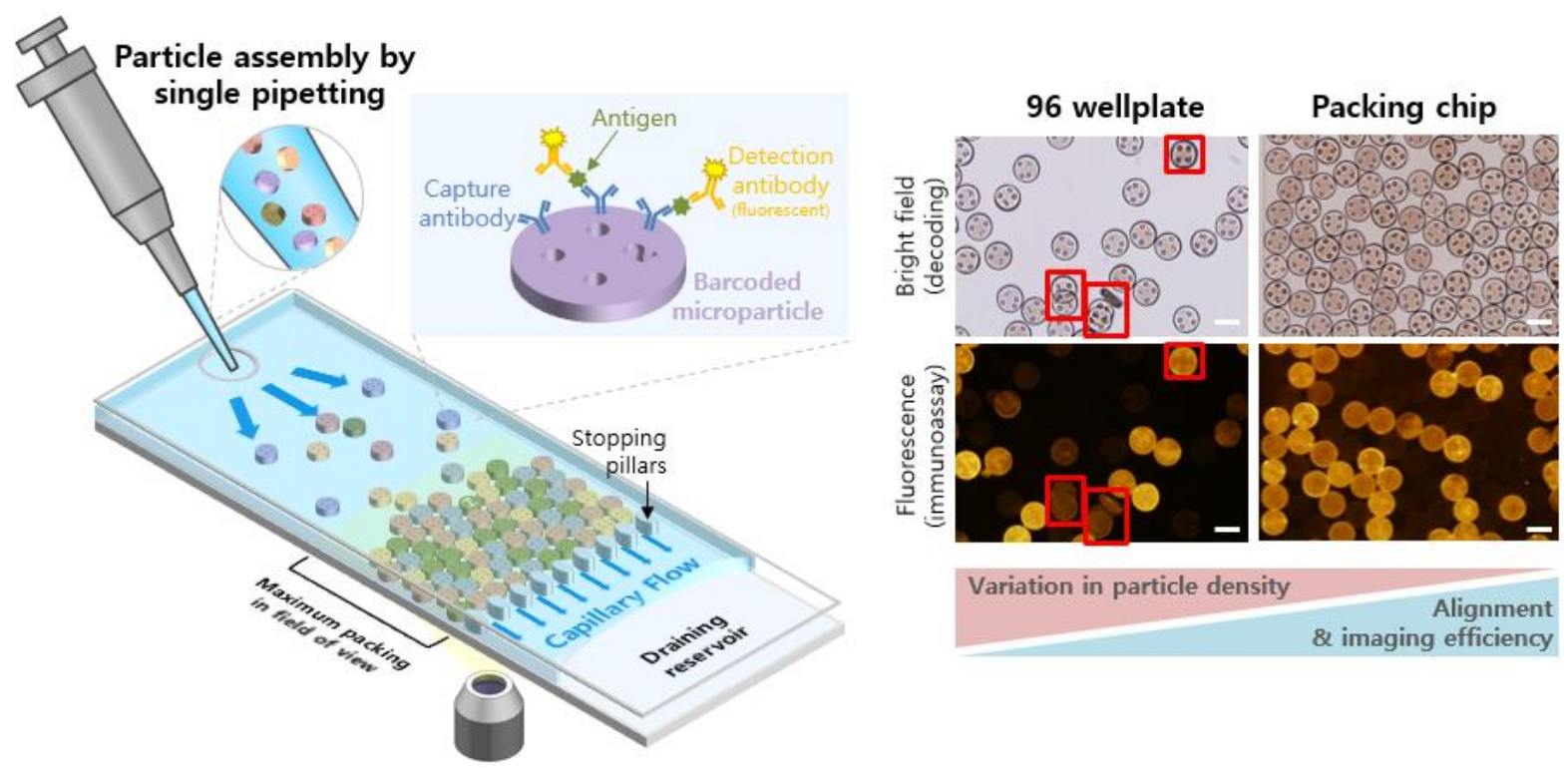

Figure 2.

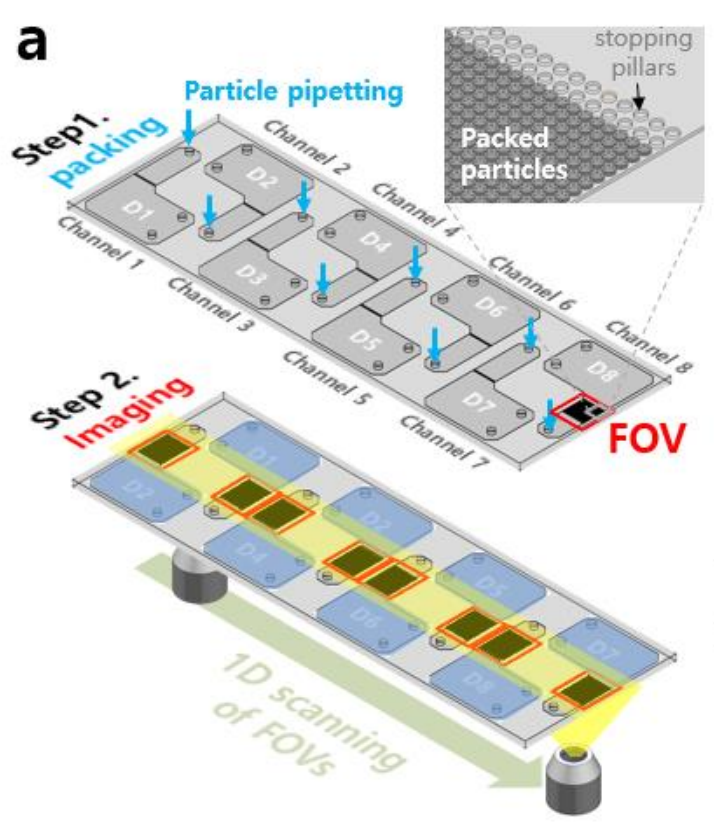

b

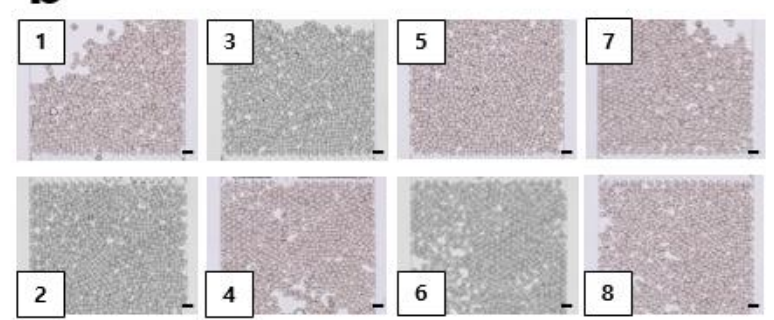

C

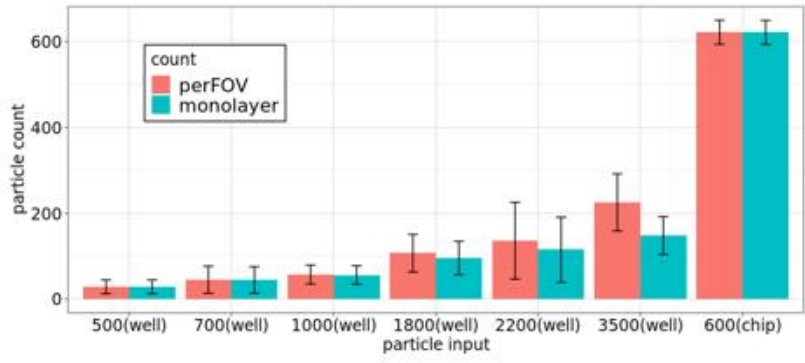


bioRxiv preprint doi: https://doi.org/10.1101/2022.01 03.474850; this version posted January 4, 2022. The copyright holder for this preprint (which was not certified by peer review) is the author/funder, who has granted bioRxiv a license to display the preprint in perpetuity. It is made available under aCC-BY-NC-ND 4.0 International license.

Figure 3.

a

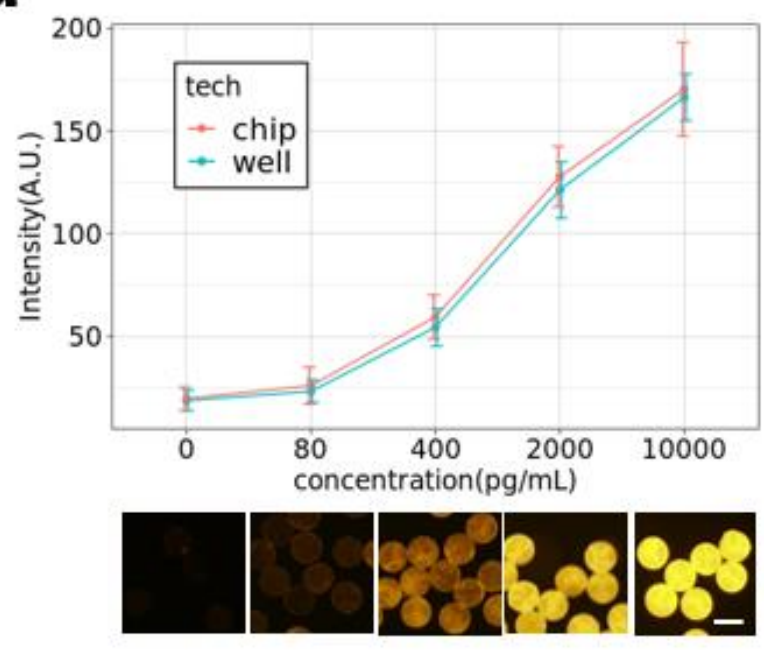

d

\section{Filter 1:}

mask

reduction

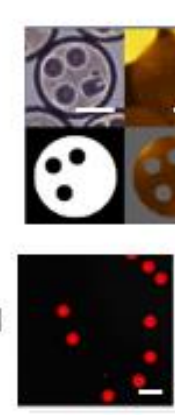

Filter 2:

background

removal

Filter 3:

particle

selection

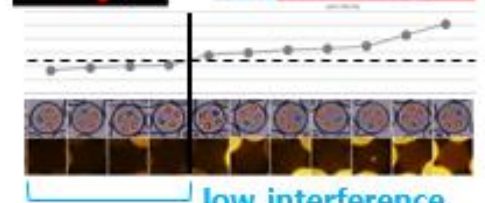

b

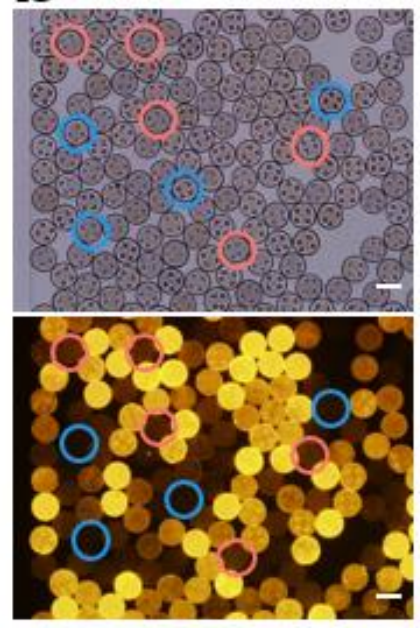

C

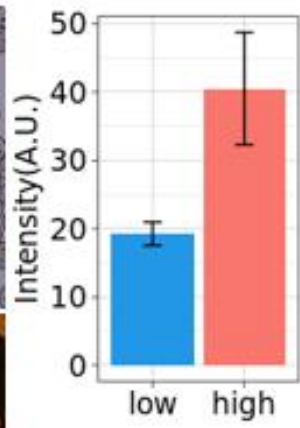

surrounded by high intensity particles

little contact with

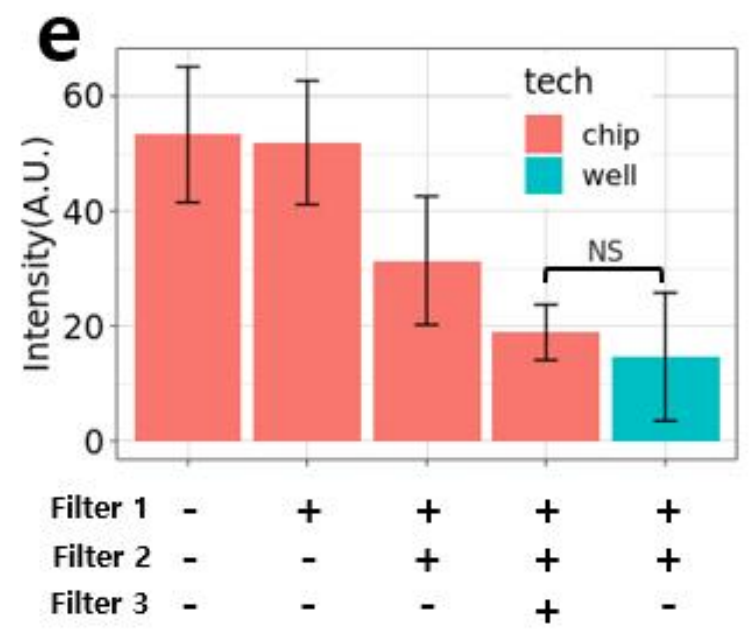


bioRxiv preprint doi: https://doi.org/10.1101/2022 01.03 474850; this version posted January 4, 2022. The copyright holder for this preprint

(which was not certified by peer review) is the author/funder, who has granted bioRxiv a license to display the preprint in perpetuity. It is made available under aCC-BY-NC-ND 4.0 International license.

Figure 4.
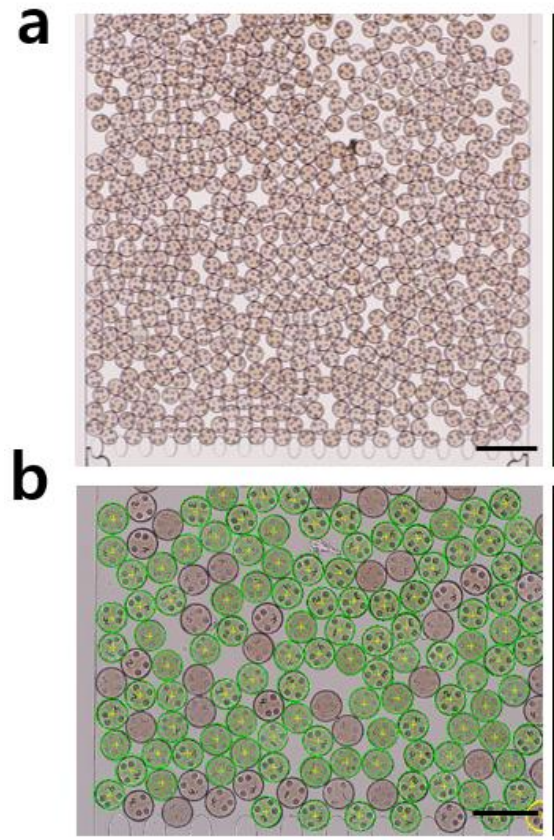
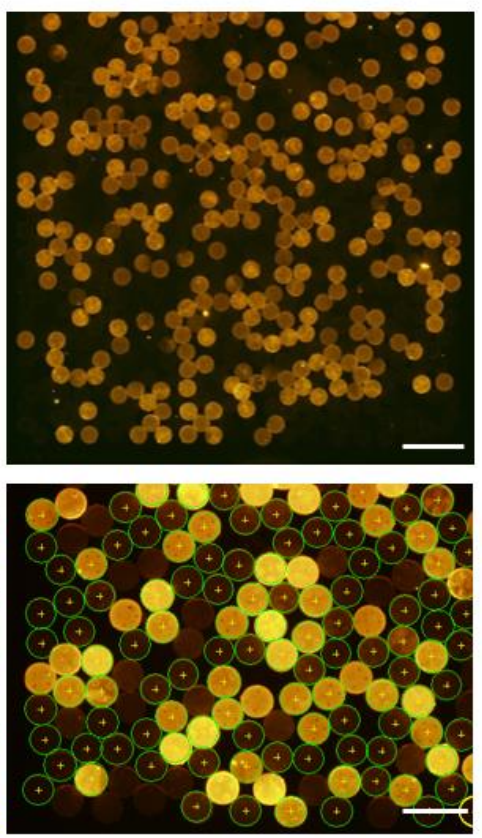
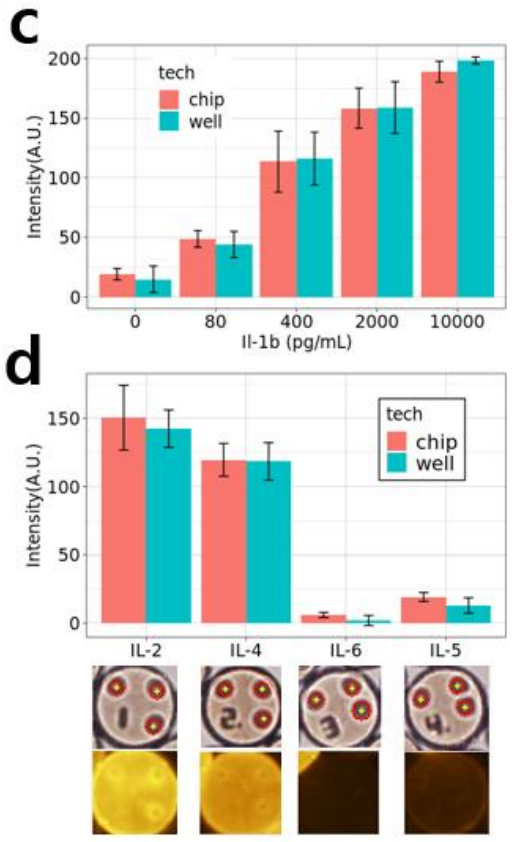

Figure 5.
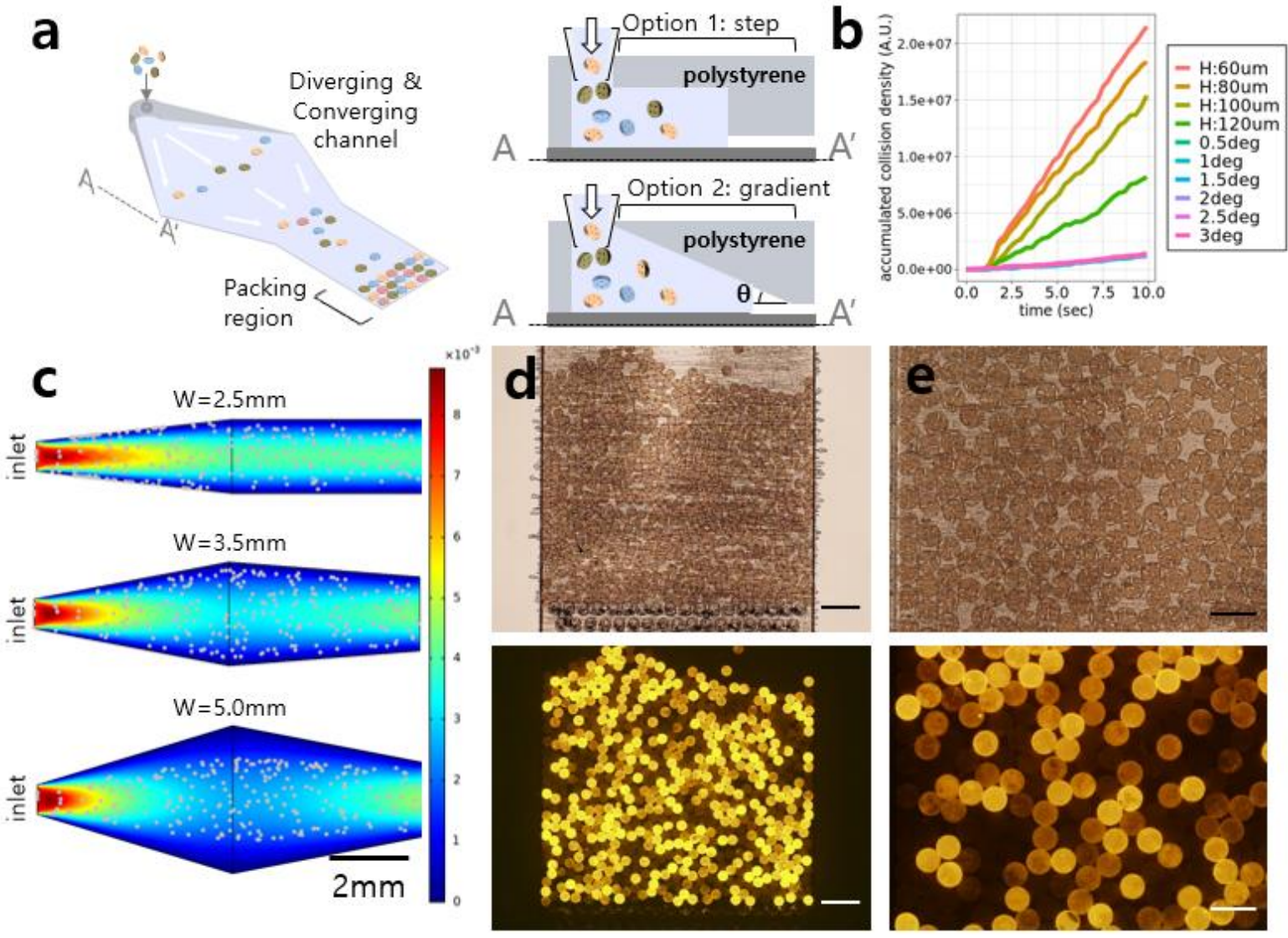